In Crescendo. Institucional. 2015; 6(1): 33-43

\title{
ESTUDIO FITOQUÍMICO Y CUANTIFICACIÓN DE FLAVONOIDES totales de las hojas de Piper Peltatum L. y Piper aduncum L. PROCEDENTES DE LA REGIÓN AMAZONAS
}

\author{
PHYTOCHEMICAL STUDY AND QUANTIFICATION OF TOTAL \\ flavonoids in the leaves of Piper Peltatum L. ANd Piper \\ ADUNCUM L. FROM AMAZONAS REGION
}

Marilú Roxana Soto Vásquez*

\section{RESUMEN}

E presente trabajo tuvo como objetivo realizar el estudio fitoquímico y determinar la concentración de flavonoides totales de las hojas de Piper peltatum L. y Piper aduncum L. procedentes de la región Amazonas. Las especies fueron recolectadas en el distrito de Río Santiago, provincia de Condorcanqui, región Amazonas. El tamizaje fitoquímico se realizó usando reactivos de coloración y precipitación. Los flavonoides totales se cuantificaron con el método de Kostennikova. Se encontró una alta diversidad de metabolitos en ambas especies, como alcaloides, triterpenos y esteroides, flavonoides, fenoles y taninos, azúcares reductores, quinonas, compuestos grasos, cumarinas y resinas; sólo se evidenció la presencia de saponinas en la especie de Piper aduncum L. El contenido de flavonoides totales en las especies de Piper peltatum L. y Piper aduncum L. fue de 1,8 $\pm 0,16$ y 2,51 \pm 0,15 g equivalentes a quercetina por cada $100 \mathrm{~g}$ de hoja seca, respectivamente; la especie de Piper aduncum L. fue la que presentó mayor concentración de flavonoides expresados como quercetina con diferencias estadísticamente significativas $(\mathrm{p}<0,05)$.

Palabras claves: Piper peltatum L., Piper aduncum L., fitoquímica, flavonoides.

\footnotetext{
* Docente de la Facultad de Farmacia y Bioquímica, Universidad Nacional de Trujillo. Correo electrónico: msoto@unitru.edu.pe
} 


\begin{abstract}
This paper aimed to make the phytochemical study and determine the concentration of total flavonoids from the leaves of Piper peltatum and Piper aduncum from the Amazon region. The species were collected from Rio Santiago district, Condorcanqui Province, Amazonas Region. The phytochemical screening was performed using staining and precipitation reagents. Total flavonoids were quantified by Kostennikova method. It was found a high diversity of metabolites in both species such as alkaloids, triterpenes and steroids, flavonoids, phenols and tannins, reducing sugars, quinones, fatty compounds, coumarins and resins, and the presence of saponins only in species of Piper aduncum L. The content of total flavonoids in species of Piper peltatum L. and L. Piper aduncum was $1.8 \pm 0.16$ and $2.51 \pm 0.15 \mathrm{~g}$ equivalents to quercetin per $100 \mathrm{~g}$ of dry leaves respectively. P. aduncum showed the highest concentration of flavonoids expressed as quercetin with statistically significant difference $(\mathrm{p}<0,05)$.
\end{abstract}

Keywords: Piper peltatum L., Piper aduncum L., phytochemistry, flavonoids. 


\section{INTRODUCCIÓN}

Desde tiempos remotos, las plantas han constituido un recurso al alcance de los seres humanos, tanto para su alimentación como para la curación de sus enfermedades (1). Aún en la actualidad muchas plantas son utilizadas en medicina y constituyen laboratorios naturales donde se biosintetiza una gran cantidad de sustancias químicas, considerándoselas como la fuente de compuestos químicos más importante que existe. Un gran porcentaje de los principios activos está comprendido dentro de los llamados productos naturales o metabolitos secundarios, que son compuestos químicos de estructuras relativamente complejas y de distribución restringida (2). Entre estos metabolitos son comunes aquellos con funciones defensivas contra insectos, bacterias y hongos, como son los alcaloides, aminoácidos no proteicos, esteroides, fenoles, flavonoides, cumarinas, quinonas, taninos y terpenoides (1).

Los flavonoides son compuestos fenólicos presentes ampliamente en la naturaleza, son responsables del buen funcionamiento de las plantas y sus beneficios para la salud humana han sido bien reconocidos en varios estudios (3). Uno de estos es su conocida capacidad antioxidante, llegándose a utilizar los flavonoides para tratar enfermedades relacionadas con procesos inflamatorios y desórdenes cardiovasculares, debido a que mejoran la circulación periférica, la movilización del colesterol y disminuyen la fragilidad capilar. También se ha documentado su actividad hepatoprotectora, antialérgica, antitrombótica, anticancerígena, antibacteriana, antifúngica, entre otras (4).

Por estas razones es necesario conocer los valores de los flavonoides presentes en recursos de importancia vegetal del país, donde encontramos especies con alto rendimiento para la elaboración de fármacos que resuelvan la problemática de salud nacional. En este sentido, la familia Piperaceae es reconocida en el Perú por presentar tres géneros y 830 especies (5). El género más rico en especies es Piper, pues estas tienen una amplia gama de actividades biológicas: antitumorales, antibióticos, antifúngicos, insecticidas; razones por las que se han hecho investigaciones fitoquímicas que han encontrado que las especies están compuestas por metabolitos de tipo flavonoide, amida, propinilfenoles, lignanos, neolignanos, kavapironas y terpenos $(6,7)$.

La especie Piper peltatum L. se conoce popularmente como "Cordoncillo" o "Santa María"; crece en sitios parcialmente cubiertos de maleza y tiene diversas aplicaciones terapéuticas como diurético, antipirético y como agente antiinflamatorio de uso interno y externo en algunas regiones de la Amazonía peruana y boliviana (8). De esta especie se ha aislado y caracterizado un gran número de compuestos químicos como amidas, lignanos, neolignanos, hidroquinonas, alcaloides, terpenos, compuestos oxigenados, y derivados del ácido benzoico (9). Por otro lado, la especie Piper aduncum L. es conocida 
popularmente como "matico". Se encuentra distribuida en Amazonas, Ayacucho, Cajamarca, Cusco, Huánuco, Junín, Loreto, Madre de Dios, Pasco, San Martín y Ucayali; sus hojas son usadas en infusión y decocción tradicionalmente para combatir las infecciones urinarias, los resfríos y la bronquitis; también como antiséptico vaginal y para curar las heridas. Se ha reportado que esta especie presenta ácidos fuertes, antocianidinas, bases cuaternarias, flavones, fenoles, piperazinas, resinas, saponinas, taninos pirogálicos y triterpenos $(9,10)$.

Las propiedades biológicas y químicas de las plantas medicinales dependen de muchos factores, entre los cuales destacan las propiedades edáficas de la región de cultivo, las condiciones del clima, la fase vegetativa, entre otras; debido a esta razón es importante el estudio de la flora en diferentes sitios, países y zonas geográficas (11). En este sentido, la presente investigación tuvo como propósito realizar el estudio fitoquímico y determinar la concentración de flavonoides totales en las hojas de Piper peltatum L. y Piper aduncum L., especies que crecen en forma silvestre en la región Amazonas.

\section{MATERIALES Y MÉTODOS}

\section{Material biológico}

Las especies de Piper peltatum L. y Piper aduncum L. fueron recolectadas en el distrito de Río Santiago (480 m. s. n. m.), provincia de Condorcanqui, región Amazonas, durante el mes de enero de 2014.

\section{Métodos}

\section{Recolección e identificación taxonómica}

La recolección de las especies se realizó por el método convencional o clásico de herborización, seleccionando el material en el campo y verificando que esté en buenas condiciones, de preferencia en las mañanas.

Un ejemplar completo de cada planta fue llevado al Herbarium Truxillense de la Universidad Nacional de Trujillo, para su identificación y posterior verificación taxonómica según el sistema filogenético de la especie.

\section{Preparación de la muestra}

Las hojas de Piper peltatum L. y Piper aduncum L. fueron seleccionadas, lavadas y desecadas en la estufa a $40{ }^{\circ} \mathrm{C}$ durante 3 días, luego fueron pulverizadas en un mortero y tamizadas por un tamiz de malla n. ${ }^{\circ} 20$. Después, las hojas tamizadas fueron almacenadas en un frasco ámbar de boca ancha. 


\section{Tamizaje fitoquímico}

El análisis del tamizaje fitoquímico se realizó según el método de Miranda \& Cuéllar (12). Se pesaron exactamente $50 \mathrm{~g}$ de cada una de las muestras y se procedió a la acción extractiva de solventes de polaridad creciente: éter etílico, etanol y agua. Luego se desarrollaron los siguientes ensayos:

El ensayo de Sudán III permite reconocer en un extracto la presencia de compuestos grasos; se considera positivo si aparecen gotas o una película coloreada de rojo en el seno del líquido o en las paredes del tubo de ensayo, respectivamente.

El ensayo de Mayer y el de Wagner permiten identificar alcaloides; se procede de la forma descrita para el ensayo de Dragendorff hasta obtener la solución ácida. Si al añadir 2 o 3 gotas de la solución reactiva de Mayer o Wagner, respectivamente, se observa opalescencia, turbidez definida y precipitado coposo, entonces se considera positiva la presencia de este tipo de metabolito. En el caso de alcaloides cuaternarios o aminoácidos libres, estos solo se encontrarán en el extracto acuoso, y para considerar su presencia debe observarse la aparición de turbidez definida o precipitado coposo en todos los casos, ya que la aparición de opalescencia puede dar un resultado falso, pues puede provenir de una extracción incompleta de bases primarias, secundarias o terciarias.

El ensayo de Baljet permite reconocer en un extracto la presencia de compuestos con agrupamiento lactónico, en particular coumarinas, aunque otros compuestos láctonicos pueden dar también positivo este ensayo. En estas condiciones se considera la presencia de esta familia de compuestos por la aparición de una coloración y un precipitado.

Para la identificación de quinonas se emplea el ensayo de Borntrager. Si la fase acuosa alcalina se colorea de rosado a rojo, el ensayo se considera positivo.

La presencia de triterpenos o esteroides se puede verificar a través del ensayo de Liebermann-Burchard, debido a que ambos tipos de productos poseen un núcleo de androstano, generalmente insaturado en el anillo B y la posición 5-6.

La presencia de aminoácidos libres o de aminas en general se comprueba a través del ensayo de Ninhidrina. Este ensayo se considera positivo cuando se desarrolla un color violáceo.

El ensayo de Shinoda permite reconocer la presencia de flavonoides en un extracto vegetal. El ensayo se considera positivo cuando el alcohol amílico se colorea de amarillo, naranja, carmelita o rojo, intensos en todos los casos.

El ensayo de antocianidinas permite identificar en los extractos la existencia en estas estructuras de secuencia C6-C3-C6 del grupo de los flavonoides. La aparición de un color rojo a marrón en la fase amílica es indicativa de un ensayo positivo. La presencia de estructuras de tipo polisacárido, que forman un coloide hidrófilo de alto índice de masa, que aumenta la densidad del agua de donde se extrae, denota la presencia de mucílagos. El ensayo de espuma permite reconocer la presencia de saponinas, tanto del tipo esteroidal como triterpénicas. Para reconocer la presencia de azúcares reductores se emplea el 
ensayo de Fehling. Este se considera positivo si la solución se colorea de rojo o aparece un precipitado rojo.

El ensayo de resina permite identificar este tipo de compuestos y se considera positivo cuando aparece un precipitado.

\section{Método espectrofotométrico para cuantificar flavonoides totales expresados como quercetina.}

Para la cuantificación de flavonoides totales se utilizó el método descrito por Kostennikova (13). Se reflujan 0,5 g de muestra $2 \mathrm{~h}$ con $20 \mathrm{ml}$ de ácido sulfúrico al $10 \%$ y $20 \mathrm{ml}$ de etanol al $50 \%$, luego se enfría y se filtra con ayuda de vacío. El residuo se lava con 30 $\mathrm{ml}$ de etanol al $50 \%$; para desecharlo finalmente. El filtrado se evapora en baño de agua hasta la mitad del volumen inicial, se enfría sobre baño de hielo durante 30 min y luego se filtra, y se lava el precipitado formado con cuatro porciones de $10 \mathrm{ml}$ de agua destilada fría $\left(10-15^{\circ} \mathrm{C}\right)$. Se elimina el filtrado, los lavados, y el residuo tanto del filtro como del recipiente, se disuelve con $70 \mathrm{ml}$ de etanol al $96 \%$, calentando previamente a $50{ }^{\circ} \mathrm{C}$; la solución se pasa a una fiola de $100 \mathrm{ml}$ y se completa el volumen con etanol al 96 \% (solución muestra). Posteriormente se leen las absorbancias a $258 \mathrm{~nm}$.

Como patrón se emplearon 0,04 g de quercetina, los cuales se disolvieron con etanol al 96 \% hasta completar un volumen de $50 \mathrm{ml}$; de esta solución se tomó $1 \mathrm{ml}$ y se diluyó a $100 \mathrm{ml}$ con etanol al $50 \%$.

El blanco consistió en una solución de etanol al 50 \%. Se realizó este procedimiento para cada especie en estudio y por sextuplicado.

Fórmula:

$$
X=\frac{A m * \operatorname{Pr} * 5}{\operatorname{Ar}} \times 100
$$

Donde:

$\mathrm{X}=$ contenido de flavonoides expresados como quercetina (\%)

Am= absorbancia de la solución muestra (nm)

$\operatorname{Pr}=$ peso de la sustancia de referencia en $\mathrm{g}$

At $=$ absorbancia de la solución de referencia $(\mathrm{nm})$ 


\section{RESULTADOS}

Tabla 1. Tamizaje fitoquímico de Piper peltatum L. y Piper aduncum L. procedentes de la región Amazonas.

\begin{tabular}{cccc}
\hline Metabolitos & Ensayos & Piper peltatum & Piper aduncum \\
\hline Alcaloides & Dragendorff & + & ++ \\
& Wagner & + & ++ \\
Triterpenos y esteroides & Mayer & + & ++ \\
Flavonoides & Lieberman Burchard & ++ & + \\
Antocianidinas & Shinoda & +++ & +++ \\
Fenoles y taninos & Antocianidina & - & \\
Saponina & Cloruro férrico & +++ & ++ \\
Azúcares reductores & Espuma & - & ++ \\
Quinonas & Fehling & ++ & ++ \\
Compuestos grasos & Borntrager & + & + \\
Catequinas & Sudán III & + & + \\
Resinas & Catequinas & - & + \\
Mucílagos & Resinas & ++ & + \\
\hline
\end{tabular}

Leyenda: (-) ausencia, (+)leve, $(++)$ Moderado, $(++)$ Abundante

Tabla 2. Cuantificación de flavanoides totales expresados como quercetina en las hojas de Piper peltatum L. y Piper aduncum L.

\begin{tabular}{|c|c|c|c|c|}
\hline Planta & $\begin{array}{c}\text { Flavonoides totales } \\
\text { expresados como rutina } \\
\text { (g/100 g de hoja desecada) }\end{array}$ & $\bar{x}$ & $D E$ & $\begin{array}{c}\text { Prueba " } \mathrm{t} \text { " } \\
\text { Student }\end{array}$ \\
\hline \multirow{6}{*}{ Piper peltatum L. } & 1,85 & \multirow{7}{*}{1,88} & \multirow{7}{*}{0,16} & \multirow{12}{*}{0,000} \\
\hline & 1,86 & & & \\
\hline & 1,87 & & & \\
\hline & 1,86 & & & \\
\hline & 1,89 & & & \\
\hline & 1,88 & & & \\
\hline \multirow{6}{*}{ Piper aduncum L. } & 2,50 & & & \\
\hline & 2,52 & \multirow{5}{*}{2,51} & \multirow{5}{*}{0,15} & \\
\hline & 2,54 & & & \\
\hline & 2,50 & & & \\
\hline & 2,51 & & & \\
\hline & 2,52 & & & \\
\hline
\end{tabular}




\section{DISCUSIÓN}

En la tabla 1 se muestran los resultados del tamizaje fitoquímico realizado a las especies de Piper peltatum L y Piper aduncum L., donde vemos la alta variabilidad de compuestos presentes en ambas especies. Destacándose entre estos: alcaloides, triterpenos y esteroides, flavonoides, fenoles y taninos, azúcares reductores, quinonas, compuestos grasos, cumarinas y resinas; se nota solo la presencia de saponinas en la especie de Piper aduncum L. Estos metabolitos coinciden con lo reportado en otros trabajos de investigación fitoquímica realizados a los géneros de Piper (14-16). Asimismo, se evidencia abundante presencia de flavonoides, fenoles y taninos en ambas especies propias de este género $(15,17)$. Estos metabolitos presentan actividades biológicas promisorias, como el eugenol (P. betle), responsable de la actividad antifúngica contra Aspergillus flavus; pseudodilapiol con actividad antimicrobiana (18). Asimismo, la presencia de compuestos fenólicos ha otorgado propiedades antioxidantes a varias especies del género Piper, tal es el caso de $P$. angustifolium $(19,20)$.

Los flavonoides están ampliamente distribuidos en el género Piper. Dentro de los principales tipos que se aislaron se encuentran las flavonas, flavanonas, chalconas y dihidrochalconas, de variada actividad biológica (antibacteriana y antifúngica); destaca también su actividad antioxidante debido a la presencia de grupos hidroxilo de tipo fenólico en la mayoría de sus estructuras químicas (21). Asimismo, se han aislado de la especie P. elongatum dos dihidrochalconas con actividad leishmanicida, la 2,6-dihidroxi-4-metoxi-dihidrochalcona y Asebogenina (22). Además, estudios realizados sobre la especie P. hispidum evidenciaron la presencia de chalconas como la $2^{\prime}$-hidroxi-3',4',6'-trimetoxichalcona, la cual mostró una importante inhibición de los amastigotes de Leishmania amazonensis ${ }^{17}$. Del mismo modo, en la especie P. crassinervium se aislaron flavonoides con núcleos flavanonona naringenina y sakuretina, los cuales presentan actividad contra cepas de hongos fitopatógenos del género Cladosporium (23). De las especies P. corniconnectivum y P. alatabaccum se aislaron metabolitos secundarios con diferentes núcleos flavonoides (flavanonas, flavonas y chalconas) con interesantes actividades larvicidas sobre Anopheles darlingi vector de la malaria en algunas regiones de la Amazonía (24). En esta misma senda, de las especies en estudio, Piper aduncum y Piper peltatum se han reportado flavonoides de tipo flavanona, flavonas y chalconas con actividades leishmanicidas, antisecretoras, citoprotectoras, antibacterianas y cicatrizantes $(25,26)$.

Para la cuantificación de flavonoides totales se utilizó el método propuesto Kostennikova. Los resultados del presente estudio indicaron que las dos especies $P$. peltatum y P. aduncum presentan valores promedios de $1,8 \pm 0,16$ y 2,51 $\pm 0,15$ g equivalentes de quercetina por cada $100 \mathrm{~g}$ de hoja seca, respectivamente. La especie de P. aduncum fue la que presentó mayor concentración de flavonoides expresados como quercetina con diferencias estadísticamente significativas $(\mathrm{p}<0.05)$ para prueba de " $\mathrm{t}$ " de Student. Estos datos se encuentran por encima de los valores reportados en estas especies ( $P$. peltatum: $1,5 \%$ y P. aduncum: 2 \%) $(27,28)$ y en otras del género Piper (P. variabile: 0,38 \%, P. oradendron: 1,57 \%, P. hispidum: 0,87 \%) (29). La cantidad de flavonoides es muy variable, 
no solamente de una especie a otra sino también dentro del mismo taxón. Esta variación se debe a mucho factores, sean climáticos, atmosféricos, edáficos y topográficos $(30,31)$.

De acuerdo con los resultados encontrados, las especies de Piper peltatum y Piper aduncum de la región Amazonas, constituyen un gran potencial para la elaboración medicinal de nuevos fármacos, así como adquieren un gran significado e importancia para las comunidades locales, que las usan de manera tradicional.

\section{CONCLUSIONES}

- Las especies de Piper peltatum L. y Piper aduncum L. presentan en las hojas una alta diversidad de metabolitos como alcaloides, triterpenos, esteroides, flavonoides, fenoles, taninos, azúcares reductores, quinonas, compuestos grasos, cumarinas y resinas; se evidenció sólo la presencia de saponinas en la especie Piper aduncum L.

- $\quad$ El contenido de flavonoides totales en las especies de Piper peltatum L. y Piper aduncum L. es de $1,8 \pm 0,16$ y 2,51 $\pm 0,15$ g que equivalen a quercetina por cada $100 \mathrm{~g}$ de hoja seca, respectivamente. La especie P. aduncum fue la que presentó mayor concentración de flavonoides expresados como quercetina.

\section{REFERENCIAS BIBLIOGRÁFICAS}

(1) Lock, O. (1994). Investigación fítoquímica; Métodos en el estudio de productos naturales. 2. ${ }^{a}$ ed. Lima: Fondo Editorial Pontificia Universidad Católica del Perú.

(2)Soto, F. (2011). Caracterización química, fitoquímica y antibacteriana in vitro de las hojas del Anacardium occidentale L. (Marañón). [Tesis para optar el grado de máster] Química-Biológica. Granma: Universidad de Granma.

(3) Pérez, G. (2003). Los flavonoides: antioxidantes o prooxidantes. Rev. Cubana Invest. Biomed. Vol. 22(1):48-57.

(4) Benavente, O. y Castillo, J. (2008). Update on uses and properties of citrus flavonoids: New findings in anticancer, cardiovascular, and anti-inflammatory activity. J. Agric. Food Chem. 56 (15), 6185-6205.

(5) León, B. (2006). Piperaceae endémicas del Perú. Rev. per. biol. Número especial 13(2): 492-563.

(6) Jara, A. (2013). Análisis fitoquímico y determinación de la actividad antioxidante del extracto etanólico de las hojas de la especie Piper imperiale (Piperaceae). Bogotá: Universidad de Ciencias Aplicadas y Ambientales. 
(7) Parmar, V., Kirpal, S. (1997). Phytochemistry of the Genus Piper. Phytochemistry. Vol. 46(4): 591-673.

(8) De Feo, V. (1991). Uso di piante ad azione antiinfiammatoria nell'Alto Ucayali, Perú Orientale. Fitoterapia. Vol. 62: 481-94.

(9) Duke, J. A., Vásquez, R. (1994). Amazonian Ethnobotanical Dictionary. Boca Ratón: CRC Press.

(10) Mejía, K., Rengifo, E. (2000). Plantas medicinales de uso popular en la Amazonía peruana. Lima: Tarea Asociación Gráfica Educativa.

(11) García, C., Martínez, A., Ortega, J., Castro, F. (2010). Componentes químicos y su relación con las actividades biológicas de algunos extractos vegetales. QuimicaViva. Vol. 2(1): 12-21.

(12) Miranda, M., Cuéllar, A. (1992). Manual de prácticas de laboratorio farmacognsoia y productos naturales. Cuba: Editorial Ciencia y Educación.

(13) Kostennikova, Z. A. (1983). UV spectrophotometric quantitative determination of flavonoid in calendula tinture. Farmatsiya. Vol. 33(6): 83-89.

(14) Giraldo, A. (2012). Estudio fitoquímico de Piperpe saresanum y Piper crassinervium (Piperaceae). [Trabajo de grado Químico Industrial]. Universidad Tecnológica de Pereira.

(15) Flores, E. (2006). Metabolitos secundarios bioactivos de especies del género Piper de la flora boliviana. [Tesis doctoral]. Universidad de la Laguna.

(16) Martínez, J. (2009). Caracterización morfológica, ecológica, genética y química de tres especies de Piper (Piper jacquemontianum, Piper donnellsmithii y Piper oradendron) con fines de conservación y mejoramiento para su aprovechamiento como nuevos recursos aromáticos y/o medicinales en Guatemala. Informe final. Guatemala: Universidad de San Carlos.

(17) Plazas, E., Cuca, L., Delgado, W. (2008). Flavonoides aislados de las inflorescencias del Piper hispidum Kunth (Piperaceae) y derivados acetilados. Revista Colombiana de Química. Vol. 37(1): 135-144.

(18) Parmar V. (1997). Phytochemistry of the genus Piper. Phytochem. Vol. 46(4): 597-673.

(19) Puertas, M., Gómez, L., Rojano, B., Saez, J. (2009). Capacidad antioxidante in vitro de fracciones de hojas de Piper peltatum L. Revista Cubana de Plantas Medicinales. Vol. 4(2):34-42.

(20) Passos et al. (2012). Evaluation of antioxidant activity and chromatographic profile of extracts from the false jaborandi (Piper aduncum). Rev. Elet. em Gestão, Educação e Tecnología Ambiental. Vol. 6(6): 1248-1260.

(21) Masuoka, C.; Ono, M.; Ito, Y.; Nohara, T. (2003). Antioxidative, Antihyaluronidase and Antityrosinase Activities of Some Constituents from the Aerial Part of Piper elongatum VAHL. Food Science and Technology Research. Vol. 9(1): 197-201. 
(22) Hermoso et al. (2003). Antileishmanial activities of dihydrochalcones from Piper elongatum and synthetic related compounds. Structural requirements for activity. Bioorganic Eamp; Medicinal Chemistry. 3975-3980.

(23) Giraldo, A. (2012). Estudio fitoquímico de Piper pesaresanum y Piper crassinervium (Piperaceae). Pereira: Universidad Tecnológica Pereira.

(24) Trindade, F. et al. (2012). Evaluation of larvicidal activity of the methanolic extracts of Piper alatabaccum branches and P. tuberculatum leaves and compounds isolated against Anopheles darlingi. Rev. bras. farmacogn. 2012; 22(5): 979-984.

(25) Puertas, M., Gómez, L., Benjamin, R., Sáez, J. (2009). Capacidad antioxidante in vitro de fracciones de hojas de Piper peltatum L. Revista Cubana de Plantas Medicinales. Vol. 14(2).

(26) Torres, E., Moreira, D., Kaplan, M., Meirelles, M., Rossi, B. (1999). Selective effect of 2',6'-dihydroxy-4'-methoxychalcone isolated from Piper aduncum on Leishmania amazonensis. Antimicrob Agents Chemother. Vol. 43(5): 1234-41.

(27) Muñoz, M. (2012). Evaluación de la actividad antiinflamatoria de extractos de Santa María (Piper peltatum) mediante el test de edema inducido en ratas (Rattus novergicus). [Tesis bioquímico farmacéutico]. Escuela Superior Politécnica de Chimborazo.

(28) Ventorim et al. (2014). Growth and production of secondary compounds in monkey-pepper (Piper aduncum L.) leaves cultivated under altered ambient light. AJCS. Vol. 8(11): 1510-1516.

(29) Cáceres, A., Cruz, S., Gaitán, I., Guerrero, K., Álvarez, L., Marroquín, M. (2012). Antioxidant activity and quantitative composition of extracts of piper species from Guatemala with potential use in natural product industry. Acta Horticulturae. Vol. 964(1): 77-84.

(30) Valares, C. (2011). Variación del metabolismo secundario en plantas debida al genotipo y al ambiente. [Doctora en Ciencias]. Universidad de Extremadura.

(31) Carrasco L. (2009). Efecto de la radiación ultravioleta-B en plantas. Idesia. Vol. 27(3): 59-76. 